\title{
Rescue Information System for Earthquake Disasters Based on MANET Emergency Communication Platform
}

\author{
Hung-Chin Jang Yao-Nan Lien Tzu-Chieh Tsai \\ Department of Computer Science, National Chengchi University \\ No. 64, Sec. 2, ZhiNan Rd., Taipei 11605, Taiwan, R.O.C. \\ +886-229387639
}

\{jang, lien, ttsai\}@cs.nccu.edu.tw

\begin{abstract}
When stricken by a catastrophic natural disaster, emergency rescue operation is very critical to many lives. Many people trapped in the disastrous areas under collapsed buildings or landslides may have a large chance to survive if they are rescued in "Golden 72 Hours". Victims rescued from the debris and evacuated from their home jammed in shelters need life support material to support their lives. They also need to communicate to each other for various reasons. However, communication systems were usually down due to various reasons. The loss of communication systems and information networks made the rescue and relief operation extremely difficult. Many people died before they got a chance to be rescued. This paper analyzes the causes that paralyzed the entire communication systems in Jiji/Taiwan Earthquake and proposes a MANET based communication platform as well as a Rescue Information System for Earthquake Disasters that can support a large number of rescue volunteers under catastrophic natural disasters.
\end{abstract}

\section{Categories and Subject Descriptors}

H.4.3 [Information Systems Applications]: Communications Applications - computer conferencing, teleconferencing, and videoconferencing.

\section{General Terms}

Algorithms, Management, Design.

\section{Keywords}

Rescue Information System, Emergency Communication, MANET.

\section{INTRODUCTION}

Almost every year, the world is stricken by numerous catastrophic natural disasters, such as earthquake, hurricane, typhoon, tsunami, etc. When stricken by a catastrophic natural disaster, such as Jiji/Taiwan Earthquake [4], SiChuan/China Earthquake [6], or Hurricane Katrina [5], emergency rescue operation is very critical to numerous lives. Many people trapped in the disastrous areas under collapsed buildings or landslides may have a large chance to survive if they are rescued in 72 hours, referred as "Golden 72 Hours". People evacuated from their home jammed in shelters need life support material to support their lives. They also need to communicate to each other for various reasons such as allocation of rescue and relief resource as well as reunion of family members. However, communication systems, fixed or mobile, were usually down due to various reasons. Rescue teams in each stricken area consists of few trained professional squads, army, police, fire fighters, and hundreds of thousands of disorganized volunteers. The loss of communication systems as well as information networks made the rescue operation extremely difficult. In Jiji Earthquake, it took ChungHwa Telecom, the largest telecommunication operator in Taiwan, 15 days of 24/7 operation to restore its mobile communication systems. Many people died before they got a chance to be rescued.

Although establishing a temporary communication network to support emergency communications and information network is one of the most urgent tasks in a disastrous rescue mission, feasible technology options are very limited in such a catastrophic disaster. We propose to use WiFi-ready notebook PCs owned by rescue volunteers themselves to construct a MANET to support such a need. Because the popularity of WiFi-ready notebook PCs is very high nowadays, this solution would be highly feasible in many countries.

The platform is designed and implemented in two phases. In the first phase, a simple MANET is implemented to support emergency information network. Rescue people, voluntary or mission-specific professional could use their own notebook PCs to construct a multi-hop ad-hoc network to form a basic wireless intranet first. In the future second phase, we will implement an "Autonomous P2P Ad-Hoc Group Communication Systems (P2Pnet)" on top of MANET. P2Pnet is used to support the communication need such as VoIP, Push-to-Talk, Instant Messaging, and mobile social network, etc. It is designed for temporary serverless infrastructureless Internet-blocked 
environments such as nature disastrous areas, battle-fields and mobile learning environments.

On top of this MANET based emergency network platform, we had implemented a Rescue Information System for Earthquake Disasters (RISED) to support rescue operations for catastrophic earthquake disasters. The system consists of Disaster Assessment Subsystem, Fastest Rescue Route Generation Subsystem, Health Care and Relief Resources Integration Subsystem, and Wounded Victim Arrangement Subsystem. The paper organization is as follows. Section two is the system analysis. Section three elaborates the disasters rescue information system we implemented. Final section is the concluding remarks.

\section{SYSTEM ANALYSIS}

\subsection{Impact of Communication System Crash}

The impact of communication system crash to the Jiji Earthquake will be presented in this section. Many people trapped in the disastrous areas under collapsed buildings or landslides may have large chance to survive if they are rescued in 72 hours, called the "Golden 72 Hour". The loss of communication system and information system created a big impact to the efficiency of rescue operation. Followings are a few painful lessons we learned from Jiji Earthquake first-handed. (One of the authors was right in a spot stricken by Jiji Earthquake.)

- In a catastrophic disaster, regular rescue teams including trained professional rescue squads, police, army, and fire fighters were far from sufficient for the emergency rescue mission. A large number of volunteers must be mobilized to participate in the rescue operation. However, without a good communication system, it is very difficult to organize and coordinate rescue volunteers.

- Transportation system was paralyzed not only by broken bridges and roads, but also by a large number of disorganized voluntary rescue vehicles.

- A large volume of rescue and relief resources were misplaced because the assessment of disasters distribution is virtually blind and inaccurate in the early hours even days after a big quake. As a consequence, the higher accessibility a stricken spot, the easier to receive external resources. Unfortunately, reallocation of resources may not be possible because of paralyzed transportation systems. In SiChuan Earthquake, some soldiers were even dropped to the disaster areas by parachutes. It is impossible to reallocate them if they were misplaced. Misplacement of rescue and relief resources may lead to catastrophic consequence. Each stricken spot may have many vulnerable survivals, such as injured people, babies and hospital patients, whose lives are highly dependent on relief resources. Misplacement of demanded medical equipments and supplies as well as life-support resources could cost numerous lives.

- Trained and skill-specific professional rescue squads were misplaced to wrong spots. For instances, a professional rescue squad specially trained and equipped for detecting survivals trapped under collapsed buildings was sent to a spot where was known having no trapped survivals.

- $\quad$ Some injured people died after being rescued from under tons of building debris because of ambulant not available or being sent to over-saturated hospitals.

- $\quad$ Streets were blocked by the collapsed buildings so that the rescue people were divided into two isolated groups. While one group was doing sound-sensitive operation (e.g. using a high sensitive sound detector to detect any human sound under debris), the group on the other side was using heavy machinery to dig the debris.

The complete list is much longer than what we mentioned above. In summary, the impact of communication system crash to a disaster could be catastrophic.

\subsection{Causes that Crashed Communication Systems}

To many people's surprise, cellular mobile communication systems that were thought highly dependable in emergency were completely wiped out in many cases. Followings are parts of causes we found in Jiji Earthquake:

- $\quad$ Base stations were crashed.

- Trunks connecting base stations to MSCs were broken almost everywhere, especially broken roads and bridges. (Trunks were laid along roads and bridges).

- $\quad$ Backup power generators were out because of fuel exhausted.

- Critical hardware equipments were down because cooling tower fell down or cooling pipes were broken.

- Cell phone ran out of battery and had no way to recharge because of power line failure or simply chargers not available.

- Communication systems were overwhelmed by extremely huge traffic. 
Threatened by so many sources of failure, it requires a miracle for a cellular mobile communication system to survive in such a catastrophic disaster, even for a robust system with $99.999 \%$ reliability. Even if there is a miracle, volunteers do not know each other and do not have time to remember large amount of phone numbers. Thus, a survived cellular communication system provides not too much help either.

\subsection{Environmental Constraints and System Requirements}

Following are the requirements for an emergency decision support system that can support a voluntary rescue operation for a catastrophic disaster.

\subsubsection{Environmental Constraints for Disastrous Spots}

- Outgoing link (Internet) is either not available or very limited.

- There is a very stringent time constraint that volunteers won't be able to use those devices that have a complicate user interface. In other words, user interface must be very simple.

- WiFi-ready notebook PCs are assumed very popular.

- Portable power generators are assumed available.

\subsubsection{Functional Requirements}

- User interface must be simple, easy to learn, and fool-proof.

- Devices do not need complicate setup procedure.

- Devices must be fault-tolerant such that misusage will not crash a device.

- Only basic functions are required, advanced features are optional.

- The system must not demand high power, must be able to recharge using a portable power generator.

Without plenty of resources and time, it is not easy to develop a system that meets all the functional requirements listed above, especially the first three. User-friendly and robustness cost a fortune to achieve. Therefore, our recommendation is to trade functionality for simplicity, developing basic functions only and giving up most advanced features.

\subsection{MANET based P2Pnet}

We propose to use WiFi-ready notebooks to construct a MANET based group communication system to support emergency communication and information network, called P2Pnet. In recent years, WiFi-ready notebook PCs that can last for several hours and universally compatible have become very popular in many areas. When stricken by a natural disaster, survivals and volunteers can use their own notebook PCs to construct a MANET to support information network as well as emergency communication systems. Compared with other options, no extra hardware cost is needed.

P2Pnet is a serverless peer-to-peer communication network based on MANET to support temporary group communication and information access. As depicted in Figure 1, some nodes may have satellite communication capability performing gateway functions so that all other nodes can access Internet through gateways if they are available. On top of MANET, there is a layer of peer-to-peer communication service to support higher level services such as Walki-Talki, Push-to-Talk, and VoIP communications.

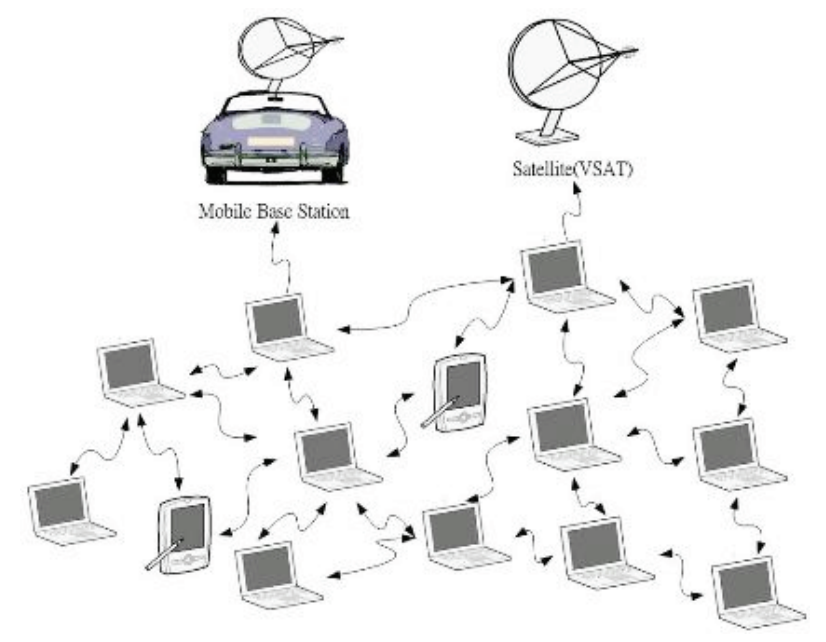

Figure 1. MANET based P2Pnet architecture.

\section{RESCUE INFORMATION SYSTEM FOR EARTHQUAKE DISASTERS}

In thin section we will elaborate on the Rescue Information System for Earthquake Disasters (RISED), which is designed to support a more efficient rescue and relief operation for catastrophic earthquake disasters. As stated above, a disaster area might lose its connection to the external world due to various factors. As a consequence, existing rescue information networks may stop functioning resulting in efficiency degradation of rescue operations. The objective of the RISED is to provide the most upto-date and accurate rescue-related information such as disaster locations, possible damages to both lives and constructions, available rescue and relief resources, the shortest way to the disaster spots, etc. The RISED will help to better manage the rescue and relief resources such that these resources can be allocated to the most needy areas in time. The decision support system is deployed on a two-tier architecture. The first-tier is the central RISED and the second-tier is the local RISED. The central RISED, deployed in the central command center (for rescue operation), is responsible for nationwise disaster assessment and resource management. A local RISED, deployed in a local command center, collects local seismic and rescue related data and uploads to the central RISED periodically at ordinary days. It can operate independently to help rescue and relief operation when the responsible region is stricken by an earthquake and loses its connection to the eternal world. If the emergency MANET groups have accesses to the Internet through some gateways that 
have external connections such as satellite communication, then the central and local RISEDs can exchange information for a more up-to-date and more accurate disaster assessment as well as a better management on the rescue operation. If, unfortunately, the emergency MANET groups have no accesses to the external world, local RISEDs can operate independently.

\subsection{ER Model of RISED}

RISED is designed to support resource and information management for the rescue mission in a catastrophic earthquake. The ER diagram of RISED is shown in Figure 2.

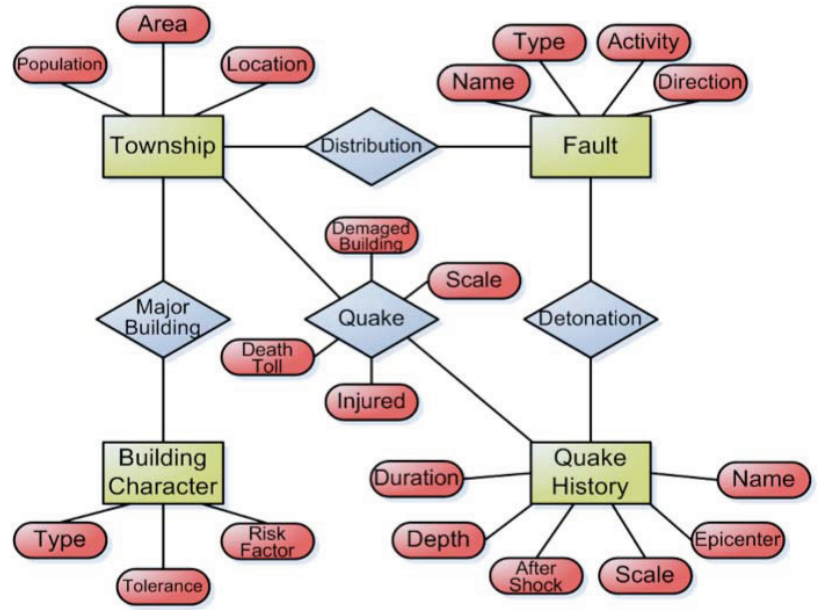

Figure 2. ER diagram of RISED.

RISED provides seismic related information to assist disaster assessment. Useful information includes seismic fault locations, population distributions, resource distributions, building characteristics, road information and quake history. Together with collected real-time disaster statistics, the information system can support a more accurate disaster assessment for a better distribution of rescue and relief resources.

\subsection{System Components of RISED}

RISED is composed of Disaster Assessment Subsystem, Fastest Rescue Route Generation Subsystem, Health Care and Relief Resources Integration Subsystem, and Wounded Victim Arrangement Subsystem (Figure 3).

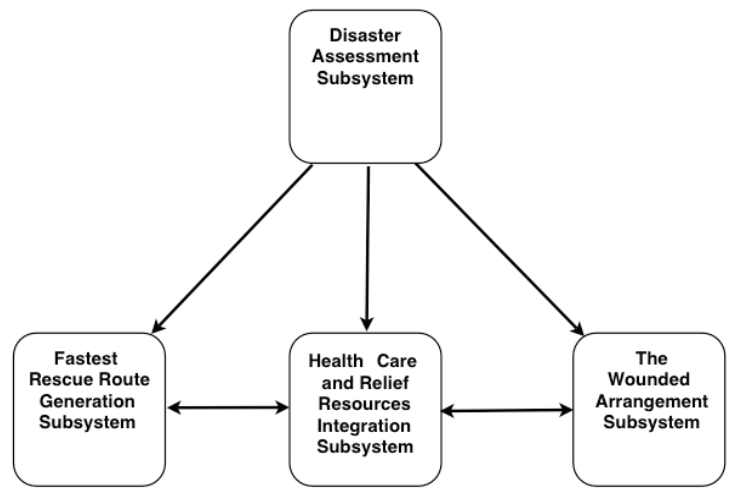

Figure 3. System components of RISED.
Disaster Assessment Subsystem: This is the most important subsystem of RISED. In the early hours of a disaster, disaster assessment may be highly inaccurate due to paralyzed communication and administration systems. To prevent rescue and relief resources from misplacement, this subsystem should collect as accurate as possible the following information: earthquake-stricken areas distribution, active faults distribution, population distribution, building profiles and other relevant information at ordinary days. As a disaster occurs, the subsystem should be able to assess the most likely occurrences of the disaster and possible damages to both people and constructions in accordance with the above collected information. The assessment will be helpful in integrating rescue and relief resource allocations, planning the fastest rescue routes to the stricken spots, and managing medical and health care resources, etc.

The input of the subsystem is epicenter and quake magnitude. The output includes the name of fault, those places belong to the same fault together with their respective quake magnitudes, populations, number of buildings, scopes of territories, degree of casualties, etc. The running steps of the subsystem are as follows:

[Step1] Find out those places belonging to the same fault

[Step2] Retrieve the data of populations, number of buildings, scopes of the territories, population density, safety coefficients of buildings, damage rate of buildings, attributes of the strata of those areas

[Step3] Derive the following values

\section{Expected damage rate of a building (of a certain sort) \\ $=$ magnitude $*(1-$ safety coefficient of the building $) *$ damage rate \\ of the buildings affected by the stratum}

Expected no. of damaged buildings

$=$ no. of buildings of a certain sort $*$ damage rate of that sort of buildings

Expected no. of the wounded victims

$=$ damage rate of a building $*$ density of buildings distribution *

rate of the wounded victims * population density

Fastest Rescue Route Generation Subsystem: When stricken by an earthquake, many roads and bridges may be broken or jammed. Traditional route planning systems are useless. The Fastest Rescue Route Generation Subsystem is able to use dynamic programming to compute the fastest rescue route to the disaster spots according to the real-time road status information. The fastest route will be used to facilitate both rescue people and resources transportation in order to reduce the number of casualties and make the rescue most effective.

The subsystem first converts the locations of road sections, road intersections and buildings into two-dimensional coordinates. Varied distances between the starting point and end points are stored into Fastest Route Database, where " 0 " represents the two points are of the same place, and "null" indicates the end point is an unreachable place. The fastest route is selected according to the following algorithm. 
Input: an undirected map, $G$

Output: a fastest route $T$, w.distance $=$ distance between $v$ and $w$

\section{begin}

Initialize the Fastest Route to empty

For (all road intersections $w$ ) do

$w \cdot$ label $=$ false $(w \cdot$ Mark $=$ true if $w$ is on the Fastest Route)

w. distance $=$ null

v.distance $=0$

while (there exists unmarked road intersections) do

Let $w$ be an unmarked road intersection

where $w$.distance is the fastest distance

$w \cdot$ Mark $=$ true

for (all route $(w, z)$ where $z$ is unmarked) do

If $(w$.distance + length $(w, z)<z$.distance $)$ then

$z$.distance $=w$.distance + length $(w, z)$

end

- Health Care and Relief Resources Integration Subsystem: This subsystem has an integrated interface to both health care and relief resources databases. These two databases altogether consist of such information as medical resources, rescue teams, shelter and life-support resources. The rescue command center can use this subsystem to retrieve the statistics of all kinds of resources distributed in different stricken areas. With these statistics, the rescue command centers can allocate and dispatch appropriate resources to the stricken areas based on their real needs.

- Wounded Victim Arrangement Subsystem: This subsystem would work with both the rescue transportation possibility and the information provided by the Health Care and Relief Resources Integration Subsystem. In the Jiji earthquake, many victims saved from the debris died in minor injuries because of inefficiency of medical resource management. For instance, they were not taken to the adequate hospitals in time resulting in fatal infections. Without a good arrangement system for wounded victims, most rescued victims were probably sent to nearby hospitals. As a consequence, some hospitals were overcrowded while others are under utilized. Victims must waste their time hopping from hospitals to hospitals.

The collaboration among the subsystems is as follows: the Disaster Assessment Subsystem is used to assess the most likely occurrences of the disaster and possible damages to both people and constructions. When an earthquake strikes, the first thing to do is to dispatch pioneering rescue teams to all potential stricken spots immediately to perform damage assessment. To save transportation time, Fastest Rescue Route Generation Subsystem is used to compute the fastest route to the stricken spots. When the assessment reports become available, Health Care and Relief Resources Integration Subsystem and Wounded Victim Arrangement Subsystem can work together to allocate medical and relief resources more accurately.

\section{CONCLUDING REMARKS}

The most important lessons we learned from numerous disasters are that mobile communication system is vulnerable and the loss of communication system and information network may have a catastrophic consequence. This paper analyzes the causes that paralyzed the entire communication systems in Jiji Earthquake and proposes a P2Pnet that uses notebook PCs to construct a MANET based emergency communication and information system. Brief system requirements and system design are presented. Finally, a prototype of Rescue Information System for Earthquake Disasters is presented.

\section{REFERENCES}

[1] Jang, H. C. and Tsai, T. C. 2001. Mobile information management system for disastrous earthquake emergency. FET Labs Journal. 3 (2001), 64-68.

[2] Lien, Y. N., Tsai, T. C., and Jang, H. C. 2000. A mobile information management system for disastrous earthquake emergency. In Proceedings of the 6th Workshop on Mobile Computing (March, 2000). 151-154.

[3] Lien, Y. N., Tsai, T. C., and Jang, H. C. 2009. A MANET based emergency communication and information system for catastrophic natural disasters. The Second International Workshop on Specialized Ad Hoc Networks and Systems (Montreal, Quebec, Canada, June 26, 2009). SAHNS 2009.

[4] Jiji Earthquake, DOI= http://140.115.123.30/921/main frame.htm, retrieved April 17, 2009.

[5] Hurricane Katrina, DOI= http://en.wikipedia.org/wiki/Hurricane Katrina, retrieved April 17, 2009.

[6] SiChuan Earthquake, DOI= http://en.wikipedia.org/wiki/2008_Sichuan_earthquake, retrieved April 17, 2009.

[7] Emergency Tolerant Cellular Systems Handling Crisis Situation, DOI= http://www.ideal-ist.net/Countries/DE/PSDE-486, retrieved April 17, 2009.

[8] Emergency Communications Systems And Equipment, DOI= http://www.gothamgazette.com/article/20040524/19/990, retrieved April 17, 2009. 